\title{
The characteristics and regularities of the climate change over the past millennium in China
}

Research on global climate change has been at the frontier of the contemporary sciences. Discussion and debate about climate warming in the recent century has attracted the attention of the world. Debate has focused on whether the greenhouse effect produced by human activities is a major factor responsible for modern climate warming and whether the warming trend will persist in the future.

The scientific argument on climate change is involved in the theories of the stochastic dynamics of a complicated climate system that are still only in early stages of development. The earth's atmosphere interacts closely and nonlinearly with oceans, land surfaces, ice and snow, and ecology. Together, they all make up a complicated nonlinear climate system. Under the influence of a changing solar system, the earth's climate has evolved in a complicated non-stationary process from its formation billions of years ago to its current status. We still know little about the characteristics, regularities, and mechanisms of climate evolution that is driven by external forces varying over time. Although the field of short-range numerical weather forecasts based on physical models is quite mature and serving society efficiently, climate methods prediction on the basis of the same physical models is less reliable and in fact no better than climate predictions based on empirical statistical methods. Therefore, at present, the ultimate goals of developing climate science and improving the performance of climate prediction are best served by strengthening basic research on climate change and deepening our understanding of the regularities and mechanisms of climate change.

In 2009, the major project "Research on tree-ring and millennium climate change in China" was implemented under the support of the National Natural Science Foundation of China. This project aims to reveal the characteristics, regularities and mechanisms of the millennium climate change in China and to establish a nonlinear dynamic method to predict climate change. In the project the tree-ring climate proxies in China and other climate data are used for diagnostic analyses, numerical modeling experiments, and nonlinear dynamic analyses.

The eight articles published in this special topic of Chinese Science Bulletin present partly preliminary results obtained by the project over the past two years. The tree-ring climate analyses and the diagnostic analyses, numerical modeling experiments, and nonlinear dynamic predictions of the millennium climate change are presented here. They reveal some characteristics and regularities of changes in temperature and precipitation in China and in East Asian monsoons over the past 1000 years. Notable conclusions are that temperatures in the Medieval Warm Period are comparable to those in the current warm period over China, and the effect of solar activity on climate cannot be neglected in any period of the millennium climate change. A method of predicting how the nonlinear dynamic system will change under the influence of external forces varying over time is preliminarily put forward. These results are important to understand the mechanisms responsible for the current climate warming and to objectively evaluate the trends of future climate change.

ZHOU XiuJi

State Key Laboratory of Severe Weather, Chinese Academy of Meteorological Sciences, Beijing 100081, China

Open Access This article is distributed under the terms of the Creative Commons Attribution License which permits any use, distribution, and reproduction in any medium, provided the original author(s) and source are credited. 